\title{
TRATAMENTO DA SUBLUXAÇÃO E LUXAÇÃO DO QUADRIL NA PARALISIA CEREBRAL EM PACIENTES TETRAPARÉTICOS ESPÁSTICOS, NIIVEL MOTOR V, ATRAVÉS DA TENOTOMIA DE ADUTORES E PSOAS
}

\author{
TREATMENT OF HIP DISPLACEMENT IN CHILDREN WITH QUADRIPLEGIC CEREBRAL \\ PALSY, GROSS MOTOR V, BY PSOAS AND ADDUCTOR RELEASE
}

Fabio Peluzo Abreu', Alexandre Zuccon², Celso $\mathrm{Cruz}^{2}$, Mauro Morais Filho ${ }^{3}$, Francisco Violante Júnior ${ }^{2}$, Paulo Kanaji $^{2}$, Daniella Neves ${ }^{2}$, Antonio Carlos Fernandes ${ }^{4}$

\section{RESUMO}

Objetivo: O objetivo do trabalho é avaliar se a tenotomia de adutores e psoas é efetiva em controlar a luxação progressiva de pacientes tetraparéticos espásticos, nível motor V. Método: Foi feito um estudo retrospectivo de 26 pacientes operados na AACD-SP com a técnica cirúrgica de tenotomia de adutores e psoas em pacientes portadores de paralisia cerebral $(\mathrm{PC})$ tetraparéticos espásticos, nível motor $\mathrm{V}$. Avaliamos o índice de Reimers pré e pós-operatório e dividimos os pacientes em dois grupos: operados abaixo de cinco anos de idade e acima de cinco anos de idade. Os dados foram levados para análise estatística. Resultados: Com um seguimento de sete anos, os dois grupos tiveram, em sua maioria dos pacientes, o índice de Reimers diminuído quando comparados ao pré-operatório. Conclusão: A tenotomia dos adutores e psoas foi efetiva em evitar a progressão da luxação em pacientes com PC tetraparéticos, nível motor V. A idade do paciente na época da cirurgia não influenciou os resultados e sim o índice de Reimers elevado no pré-operatório e quadris assimétricos.

Descritores - Paralisia Cerebral; Luxação do Quadril; Segmentos

\section{ABSTRACT}

Objective: To assess if adductor and psoas release is effective in prevent hip displacement in quadriplegic Cerebral Palsy pacients, Gross motor V. Methods: A retrospective study with 26 patients who had psoas and adductor release was analyzed. Reimers Index before and after surgery was analysed. We divided the patients into two groups: surgery made in patients above five yars and after five years of age. Results: With a follow up of seven years, most of the patients of both groups had Reimers Index controlled by the surgery proposed. Conclusion: Adductor and psoas release was effective in controlled hip displacement in quadriplegic Cerebral Palsy patients, Gross Motor V. The age at the time of the surgery does not influence in the result.Reimers Index up to $70 \%$ and asymmetric hip not had good results.

Keywords - Cerebral Palsy; Hip Dislocation; Follow-Up Studies

\section{INTRODUÇÃO}

A subluxação e a luxação do quadril na paralisia cerebral é um problema grave e comum, principalmente nos não deambuladores, nos quais a prevalência pode chegar a $80 \%$ dos $\operatorname{casos}^{(1,2)}$. Uma das possíveis causas deste problema é a espasticidade dos adutores e flexores do quadril, que perante a um quadro de extensores e abdutores mais fracos provoca um desequilíbrio de forças musculares com extrusão progressiva da articulação coxofemoral. Essa condição pode ser dolorosa, além de gerar limitações para o posicionamento e a realização da higiene perineal ${ }^{(3,4)}$.

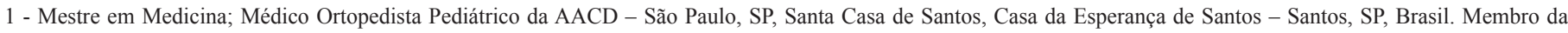
Sociedade Brasileira de Ortopedia Pediátrica.

2 - Médico, Ortopedista Pediátrico da AACD - São Paulo, SP. Membro da Sociedade Brasileira de Ortopedia Pediátrica.

3 - Mestre em Medicina, Médico Ortopedista Pediátrico e Diretor Clínico da AACD - São Paulo, SP, Brasil.

4 - Mestre em Medicina; Médico Ortopedista Pediátrico da AACD - São Paulo, SP. Membro da Sociedade Brasileira de Ortopedia Pediátrica.

Trabalho realizado na AACD - São Paulo, SP.

Correspondência: Rua Doutor Luís Suplicy, 49 - 11055-330 - Santos, SP. E-mail: fabiopeluzo@uol.com.br

Trabalho recebido para publicação: 24/08/2011, aceito para publicação: 31/08/2011.

Os autores declaram inexistência de conflito de interesses na realização deste trabalho / The authors declare that there was no conflict of interest in conducting this work 
Miller e $\mathrm{Bagg}^{(3)}$ correlacionaram a idade e o risco de subluxação em pacientes com paralisia cerebral e observaram que a chance de progressão é a mesma em todas as faixas etárias ate os 18 anos de idade. Após essa idade, quadris com extrusão até $29 \%$ tendem a se manter estáveis; enquanto que aqueles com subluxação maior que $60 \%$ devem progredir mesmo após os 18 anos.

Medidas a fim de evitar essa subluxação estão descritas na literatura e incluem observação, aparelhos para manter o quadril reduzido, fisioterapia, bloqueios neuroquímicos e cirurgias que visam equilibrar as forças musculares $^{(1)}$.

A realização de tenotomia dos adutores de quadril e psoas no pequeno trocânter tem indicação clássica nos casos detectados precocemente, nas quais a extrusão da cabeça femoral é leve ${ }^{(5)}$.

Com isso, o objetivo deste estudo foi avaliar, em uma série consecutiva de casos, se a tenotomia dos adutores de quadril e do psoas no pequeno trocânter foi efetiva para controle da subluxação progressiva em pacientes com paralisia cerebral tipo tetraparesia espástica, do nível motor $\mathrm{V}$.

\section{CASUÍSTICA E MÉTODOS}

Foi realizado um estudo retrospectivo através da análise de prontuários da AACD e dos pacientes submetidos à tenotomia do psoas e adutores de quadril em nossa instituição de janeiro de 2000 a janeiro de 2001 , com o objetivo de controlar a subluxação progressiva. O diagnóstico de paralisia cerebral tipo tetraparesia espástica e a completa documentação radiográfica foram considerados como critérios de inclusão.

O procedimento cirúrgico foi realizado pela equipe de ortopedia pediátrica da instituição através da mesma técnica padronizada, ou seja, a tenotomia do adutor longo, miotomia do grácil e a tenotomia do psoas, ao nível do pequeno trocânter, em todos os pacientes, com o objetivo de proporcionar uma abdução maior ou igual a $45^{\circ}$. Em todos os pacientes os procedimentos foram realizados bilateralmente e não houve associação com procedimentos ósseos ao nível do quadril. No pós-operatório foi colocada uma tala inguinomaleolar e mantida em abdução em membros inferiores de $45^{\circ}$ por três semanas.

Os seguintes parâmetros foram analisados:

- O índice de Reimers pré-operatório;

- O índice de Reimers atual;

- Idade na ocasião da cirurgia;
- Tempo de seguimento; e

- Complicações no pós-operatório.

Com relação à progressão da subluxação, foi considerado um bom resultado a presença de um índice de Reimers igual ou menor ao pré-operatório na última avaliação.

Dois grupos foram criados de acordo com a idade dos pacientes na cirurgia. No grupo A, foram incluídos aqueles que receberam a tenotomia de psoas e adutores antes dos cinco anos de idade $(n=12)$, enquanto que no grupo B $(n=14)$ foram agrupados os que foram operados após os cinco anos. O índice de Reimers médio pré e pós-operatório foi comparado entre os grupos e submetido à análise estatística com a aplicação do teste de Wilcoxon com nível de nulidade de $5 \%$ ou 0,05 .

\section{RESULTADOS}

O tempo médio de seguimento (tempo decorrido entre o ato cirúrgico e a última radiografia e consulta do paciente) no estudo foi de sete anos. Em 20 dos 26 pacientes estudados, o índice de Reimers manteve-se com o mesmo valor ou diminuiu em relação à medida pré-operatória. $\mathrm{O}$ índice de Reimers médio passou de $43 \%$ para $24 \%$ a direita, e de $41 \%$ para $33 \%$ a esquerda. Como complicação pós-operatória, dois pacientes apresentaram deiscência de sutura em região dos adutores, evoluindo com cicatrização por segunda intenção sem intercorrências.

Em 12 pacientes, o procedimento foi feito abaixo dos cinco anos (grupo A) e o índice de Reimers, que era de $46 \%$ em média, passou a ser de $34 \%$ (Tabela 1 ). Apesar da redução, esta diferença não foi significativa $(\mathrm{z}$ calculado $=1,93 / \mathrm{z}$ crítico $=1,96)$. Em 14 pacientes, o procedimento foi feito acima dos cinco anos (grupo B) e a média do índice de Reimers pré era de $40 \%$ e foi reduzida para $26 \%$ (Tabela 1) que foi considerada estatisticamente significativa.

Tabela 1 - Índice de Reimers médio pré e pós-operatório nos dois grupos estudados.

\begin{tabular}{c|c|c}
\hline $\begin{array}{c}\text { Idade na época da } \\
\text { cirurgia }\end{array}$ & $\begin{array}{c}\text { Índice de Reimers } \\
\text { médio } \\
\text { pré-operatório }\end{array}$ & $\begin{array}{c}\text { Índice de Reimers } \\
\text { médio } \\
\text { pós-operatório }\end{array}$ \\
\hline Abaixo de cinco anos & $46 \%$ & $34 \%$ \\
\hline Acima de cinco anos & $40 \%$ & $26 \%$ \\
\hline
\end{tabular}


$\mathrm{Na}$ amostra estudada, oito quadris apresentavam índice de Reimers $>70 \%$ e todos eles apresentaram resultados insatisfatórios após a realização do alongamento dos adutores e psoas. Por outro lado, 28 quadris apresentavam índice de Reimers abaixo de $40 \%$ e, desses, 26 mantiveram ou diminuíram o índice de Reimers.

Oito pacientes apresentaram quadris simétricos (mesmo valor do índice de Reimers entre o lado direito e o lado esquerdo) no pré-operatório e com índice de Reimers abaixo de $60 \%$ e, desses, apenas dois obtiveram progressão da deformidade.

Vinte e cinco quadris apresentavam índice de Reimers normal na época da cirurgia (IR menor que 33\%) e apenas um evoluiu com aumento do índice de Reimers, necessitando de reconstrução óssea.

\section{DISCUSSÃO}

Analisando os nossos resultados, dividimos para análise estatística os pacientes em dois grupos: menores que cinco anos (grupo A) e maiores que cinco anos (grupo B). No grupo A, o resultado não foi significativo $(\mathrm{z}$ calculado $=1,93 ; \mathrm{z}$ crítico $=1,96), \mathrm{e}$, no grupo $\mathrm{B}, \mathrm{o}$ resultado encontrado foi significativo $(\mathrm{z}$ calculado $=$ 3,57 ; $\mathrm{z}$ crítico $=1,96$ ) pela análise estatística. Ao analisarmos esses dados, de um total de 12 pacientes do grupo A (menores que cinco anos), só três pacientes tiveram o índice de Reimers aumentado. Apesar de o número estatístico ser quase significativo, os valores reais mostraram $75 \%$ de bons resultados (nove pacientes) no grupo menor que cinco anos.

Analisando-se o grupo B (maiores que cinco anos), de um total de 14 pacientes, um paciente teve aumento do índice de Reimers; porém, em cinco, o índice de Reimers não mudou, e, em oito, houve redução do seu valor. No grupo A, oito pacientes (66\%) também tiveram diminuição do índice de Reimers.

Estudos na literatura divergem em relação ao índice de Reimers e indicação do procedimento em partes moles a fim de manter o quadril reduzido. Onimus et $a l^{(2)}$ relataram bons resultados em 11 pacientes de um total de 20 pacientes com índice de Reimers menor que $33 \%$. Nosso estudo mostrou que 11 dos 20 pacientes com bons resultados apresentaram índice de Reimers acima de $40 \%$ e se beneficiaram com a cirurgia.

Turker e Lee ${ }^{(4)}$, em seu estudo, mostraram que 50\% dos pacientes submetidos ao procedimento em partes moles apresentaram nova reluxação ou luxação. Em nosso estudo, apenas $23 \%$ dos pacientes apresentaram reluxação ou progressão da migração. Todos pacientes que estavam com quadris luxados mantiveram-se com os quadris luxados.

Com relação aos pacientes que assumiam o ortostatismo (quatro pacientes faziam uso de parapódio), todos apresentaram bons resultados, o que vai de acordo com a literatura. Miller e $\mathrm{Bagg}^{(3)}$ concluíram, em seu estudo, que dois fatores são importantes para bom prognóstico: pacientes deambuladores e pacientes com menor envolvimento motor. Nesse mesmo estudo, os autores referem que a idade na época da cirurgia não influenciava nos resultados, o que vai de acordo com nosso estudo; porém, nossos pacientes não eram deambuladores e eram mais gravemente acometidos.

Não há consenso na literatura com relação à idade e à época da cirurgia. Reimers ${ }^{(6)}$ observou melhores resultados quando a cirurgia era realizada antes dos quatro anos de idade. Onimus et $a l^{(2)}$ reportaram 11 bons resultados em um total de 12 pacientes quando a cirurgia foi realizada abaixo dos quatro anos e com índice de migração menor do que $30 \%$. Turker e Lee ${ }^{(4)}$ não evidenciaram relação entre a idade na época da cirurgia e o resultado pós-cirúrgico. Em nosso estudo, essa relação também não foi evidenciada estatisticamente, porém a casuística foi pequena. Sabemos que a chance de remodelação do acetábulo e a melhora no índice de Reimers são menores quando a cirurgia é realizada após os oito anos, porém o melhor equilíbrio muscular com o tratamento cirúrgico evita a progressão da luxação mesmo em pacientes maduros, e essa não progressão pode ser considerada como bom resultado. Miller e $\mathrm{Bagg}^{(3)}$ afirmam que pacientes com índice de Reimers acima de 30\% apresentam 23\% de progressão mesmo após a maturidade esquelética, o que pode justificar o procedimento em pacientes adultos e com subluxação de quadris.

A progressão da luxação em nossos pacientes aconteceu naqueles gravemente acometidos e com deformidade progressiva na coluna, gerando obliquidade da pelve e luxação. A associação de quadril em ventania, obliquidade pélvica e escoliose com a luxação do quadril tem sido alvo de diversos estudos na literatura ${ }^{(1,7,8)}$. Há concordância de que o quadril em ventania favorece a luxação de quadril e é de difícil tratamento mesmo com tenotomias múltiplas. A obliquidade pélvica e a escoliose também estão associadas à luxação de quadril em pacientes com desequilíbrio muscular. Cornell et $a l^{(5)}$, em um estudo com pacientes portadores de paralisia cerebral, mostrou que o tratamento através da 
tenotomia de adutores e psoas foi efetivo em manter o índice de Reimers nos parâmetros pré-operatórios, evitando a progressão da luxação na maioria dos pacientes. Neste estudo, a idade do paciente na época da cirurgia não influenciou no resultado e sim o índice de migração do pré-operatório. Reimers ${ }^{(6)}$ não realizava a tenotomia do psoas de rotina, só em pacientes que apresentavam deformidade em flexão do quadril. Ele acreditava que a tenotomia do psoas não tem efeito sobre o índice de migração. Kalen e Bleck ${ }^{(1)}$ também fazem a tenotomia do psoas só em pacientes que apresentam contratura em flexão do quadril. Eles obtiveram $72 \%$ de bons resultados em pacientes submetidos à tenotomia do psoas em comparação com $64 \%$ de pacientes não submetidos a este procedimento. Onimus et $a l^{(2)}$ fizeram a tenotomia do psoas em todos os pacientes e mostraram um resultado de $65 \%$ em 40 quadris. Em nosso estudo, a tenotomia do psoas foi feita em todos os pacientes e os resultados foram satisfatórios em comparação ao estudo de Onimus. Acreditamos que a força flexora do quadril é importante na contribuição do índice de migração para progressão da luxação e a utilizamos em todos os pacientes não deambuladores submetidos ao tratamento cirúrgico.

\section{CONCLUSÃO}

A tenotomia do psoas e dos adutores foi eficaz na redução da migração dos quadris em pacientes portadores de paralisia cerebral espásticos e tetraparéticos. Quadris luxados e com índice de Reimers elevados (acima de $70 \%$ ) tendem a se manter luxados. Pacientes com índice de Reimers de 50 e $60 \%$ apresentam bons resultados quando os quadris são simétricos (mesmo valor do índice de Reimers do quadril direito e do quadril esquerdo).

Índices de Reimers abaixo de $40 \%$ apresentam bons resultados com os procedimentos relatados. A idade à época da cirurgia não influenciou os resultados.

\section{REFERÊNCIAS}

1. Kalen V, Bleck EE. Prevention of spastic paralytic dislocation of the hip. Dev Med Child Neurol. 1985;27(1):17-24.

2. Onimus M, Allamel G, Manzone P, Laurain JM. Prevention of hip dislocation in cerebral palsy by early psoas and adductors tenotomies. J Pediatr Orthop. 1991;11(4):432-5.

3. Miller F, Bagg MR. Age and migration percentage as risk factors for progression in spastic hip disease. Dev Med Child Neurol. 1995;37(5):449-55.

4. Turker RJ, Lee R. Adductor tenotomies in children with quadriplegic cerebral palsy: longer term follow-up. J Pediatr Orthop. 2000;20(3):370-4.
5. Cornell MS, Hastrick NC, Boyd R, Baird G, Spencer JD. The hip in children with cerebral palsy. Predicty the outcome of soft tissue surgery. Clin Orthop Relat Res, 1997;( 340 ):165 -71.

6. Reimers $\mathrm{J}$. The stability of the hip in children. A radiological study of the results of muscle surgery in cerebral palsy. Acta Orthop Scand Suppl. 1980;184:1-100.

7. Abel MF, Blanco JS, Pavlovich L, Damiano DL. Asymmetric hip deformity and subluxation in cerebral palsy: an analysis of surgical treatment. J Pediatr Orthop. 1999;19(4):479-85.

8. Lonstein JE, Beck K. Hip dislocation and subluxation in cerebral palsy. J Pediatr Orthop. 1986;6(5):521-6. 